\title{
Operator relations and chemical effects on chiral dynamics in QCD
}

\author{
Rajarshi Ray ${ }^{* \dagger}$ \\ Saha Inst of Nuclear Physics, Kolkata,India \\ E-mail: rajarshi.rayesaha.ac.in
}

\section{Sourendu Gupta}

Tata Institute of Fundamental Research,Mumbai,India

E-mail: sgupta@tifr.res.in

\begin{abstract}
We discuss effects of chemical potential on the dynamics of chiral transition by the Taylor expansion method in lattice QCD. Operators and their Taylor expansions which characterise the chiral dynamics are related through Maxwell's relations and Chiral Ward identities. This reduces the computation to just measuring the renormalized expectation values of the chiral condensate and its Taylor coefficients. The various relations can then be used to extract information of the operators responsible for the characteristics of the chiral dynamics.
\end{abstract}

XXIIIrd International Symposium on Lattice Field Theory

25-30 July 2005

Trinity College, Dublin, Ireland

\footnotetext{
* Speaker.

${ }^{\dagger}$ More details of the work is reported in ref. [7]
} 


\section{Introduction}

Direct lattice simulations at non-zero chemical potential are possible only along certain subspaces of the full parameter space, such as $\operatorname{Re} \mu_{f}=0$ or $\mu_{u}=-\mu_{d}$. However, Taylor expansions around $\mu_{f}=0$ have proved to be useful in studying the physics for general $\mu_{f}$. Here we discuss the two flavour case $f \in\{u, d\}$ and with degenerate quark masses $m_{u}=m_{d}=m$. We shall freely go from the parameter space expressed in terms of $\mu_{u}$ and $\mu_{d}$ to the isoscalar $\mu_{0}=\left(\mu_{u}+\mu_{d}\right) / 2$ and the isovector $\mu_{3}=\left(\mu_{u}-\mu_{d}\right) / 2$ chemical potentials. These are simply related to the baryon and electric charge chemical potentials. Since we perform Taylor expansions around the point $\mu_{f}=0$, all expectation values are computable by the standard methods of lattice gauge theory.

Various operators related to chiral dynamics in QCD are connected among themselves via chiral Ward identities and Maxwell relations, such that there are only few independent Taylor coefficients. For us the three independent parameters of interest in the chiral sector are the single linear response coefficient of the chiral condensate to $\mu$, and two quadratic response coefficients (QRCs). The linear coefficient vanishes by symmetry in an expansion around $\mu=0$. Thus, the two chiral QRCs encapsulate the physics arising from the influence of baryon dynamics due to $\mu \neq 0$ on chiral fluctuations.

\section{Taylor Expansion}

\subsection{Chiral condensate}

Here we consider only the isoscalar condensate $C_{S}\left(m, T, \mu_{u}, \mu_{d}\right)=\frac{1}{2}[\langle\bar{u} u\rangle+\langle\bar{d} d\rangle]$, which can also be written as, $C_{S}\left(m, T, \mu_{u}, \mu_{d}\right)=\left.\frac{1}{2 V_{4}}\left(\frac{\partial \log Z}{\partial m_{u}}+\frac{\partial \log Z}{\partial m_{d}}\right)\right|_{m_{u}=m_{d}=m}$ where $V_{4}$ is the 4-volume of the lattice. In our present computations the volumes are taken large enough, but with non-zero quark masses. Since we work with staggered quarks, the mass renormalization is multiplicative, and so are the renormalization of the condensate and its Taylor coefficients. We are interested only on the variation of the condensate with $\mu$ at constant $T$, so we simply work with either of the ratios

$$
\frac{C_{S}\left(m_{R}, T, \mu_{u}, \mu_{d} ; a\right)}{C_{S}\left(m_{R}, 0,0,0 ; a\right)} \quad \text { (Z scheme) } \quad \text { or } \quad \frac{C_{S}\left(m_{R}, T, \mu_{u}, \mu_{d} ; a\right)}{C_{S}\left(m_{R}, T, 0,0 ; a\right)} \quad \text { (T scheme). }
$$

The computations have to be performed at fixed renormalized mass $m_{R}$. One expects the T scheme to be undefined for $T>T_{c}$ when $m_{R}=0$, since the chiral condensate then vanishes. We perform a formal expansion here which will later be renormalized in either of these schemes.

$$
\begin{aligned}
& C_{S}\left(m, T, \mu_{0}\right)=C_{S}^{0}+\left(C_{S}^{20}+C_{S}^{11}\right) \frac{\mu_{0}^{2}}{2}+\cdots \\
& C_{S}\left(m, T, \mu_{3}\right)=C_{S}^{0}+\left(C_{S}^{20}-C_{S}^{11}\right) \frac{\mu_{3}^{2}}{2}+\cdots
\end{aligned}
$$

where $C_{S}^{20}$ and $C_{S}^{11}$ are respectively the diagonal and off-diagonal coefficients.

\subsection{Chiral Ward identities}

Chiral Ward identities are consequences of certain operator equalities which follow from chiral symmetries. The prototypical chiral Ward identity is $C_{S}(T, \mu)=m \chi_{\pi}(T, \mu)$, where $\chi_{\pi}$ is the 
pseudo-scalar susceptibility. Taylor expansions of both sides of this identity can then be equated term by term. A second chiral Ward identity [1] relates the isovector scalar susceptibility to the mass derivative of the condensate: $\partial C_{S} / \partial m=-\chi_{\varepsilon}$.

We show later that in the continuum limit of the high temperature phase the second derivatives of the condensate with respect to the chemical potential vanish. As a result, the the pion susceptibilities are insensitive to chemical potential. Also, in this phase, symmetry arguments show that $\chi_{\pi}=\chi_{\varepsilon}$ [2]. As a result, the scalar susceptibility is also independent of the isoscalar chemical potential, at least to quadratic order. Below $T_{c}$ this chain of logic does not hold. The scalar susceptibility is interesting because of speculation about the massless modes at the critical end point [3]. We will discuss it at greater length elsewhere.

\subsection{Maxwell relations}

A Maxwell relation is the equality of two distinct physical interpretations of a mixed derivative obtained by interchanging the order of the derivatives. From the Taylor expansion of the chiral condensate in eq. (2.2) we can find Maxwell relations with the change of quark number susceptibilities (QNS) with the quark mass. The leading order relation $\frac{\partial C_{S}}{\partial \mu}=\frac{\partial n}{\partial m}$ was first noted in [4]. It is trivially true at $\mu=0$ since the first derivative on the left vanishes, as does $n$ for all quark masses. The second derivatives give two non-trivial Maxwell relations and consequent relations using the chiral Ward identities discussed earlier,

$$
\begin{aligned}
& C_{S}^{20}=\frac{\partial^{2} C_{S}}{\partial \mu_{u}^{2}}=m \frac{\partial^{2} \chi_{\pi}}{\partial \mu_{u}^{2}}=\frac{\partial \chi_{u u}}{\partial m}, \\
& C_{S}^{11}=\frac{\partial^{2} C_{S}}{\partial \mu_{u} \partial \mu_{d}}=m \frac{\partial^{2} \chi_{\pi}}{\partial \mu_{u} \partial \mu_{d}}=\frac{\partial \chi_{u d}}{\partial m} .
\end{aligned}
$$

Here, as a byproduct of our computation of the renormalized chiral condensate, we shall give the continuum limit of the derivative of the susceptibility. Also, the relative rates of strange and light quark production in heavy-ion collisions i.e. the Wroblewski parameter on the lattice [5] is

$$
\lambda_{s}=\frac{\chi_{s s}}{\chi_{u u}}
$$

with obvious extensions to the production rates of heavier quarks. Since it is hard to perform lattice computations at realistic values of light quark masses due to constraints of computer time, one can lighten the computational burden by using a Taylor series for the mass dependence of the susceptibilities utilizing the above Maxwell relation. Then one can compute $\lambda_{s}$ at some reasonably light quark mass, corresponding to, say, the pion mass being two to three times heavier than in the real world, and extrapolate to the physical quark mass values using the Maxwell relation. Extrapolating the results of our measurements at heavier quark masses to the realistic values we find the change in $\lambda_{s}<1 \%$.

\section{Results}

\subsection{Continuum limit in quenched QCD}

\subsection{1 $T>T_{c}$}

We used stored gauge configurations from the study in [6] for our measurements. These were 
obtained on $N_{t}=4,8,10,12$ and 14 lattices for temperatures $T=1.5 T_{c}, 2 T_{c}$ and $3 T_{c}$ respectively.

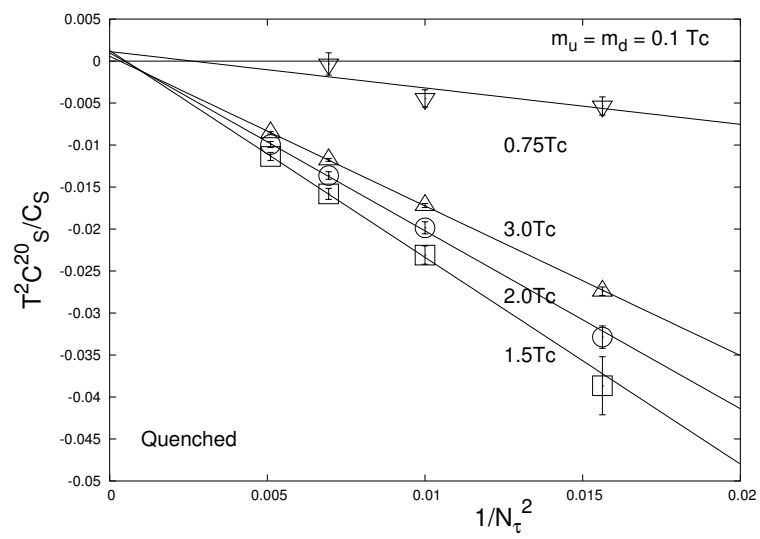

Figure 1: The diagonal $\mathrm{QRC}$ in the $\mathrm{T}$ scheme, at fixed $T / T_{c}=1.5$ holding the renormalized quark mass fixed by fixing the bare mass to be $m / T_{c}=0.1$. The lines show the continuum extrapolation.

The diagonal QRC in the T scheme is shown in Figure 1. It is clear that there is significant $\mu$-dependence on coarser lattices. However, this Taylor coefficient vanishes at the $99 \%$ confidence limit on extrapolation to the continuum. The identities in eq. (2.3) then imply that $\chi_{u u}$ is insensitive to changes in the quark mass and $\chi_{\pi}$ is insensitive to $\mu$ in this range of temperatures. Since $\chi_{u u}$ agrees with a perturbative evaluation for $T \geq 1.5 T_{c}$, its insensitivity to $m$ can be understood from the fact that the effective infrared cutoff is given by the Matsubara frequency $\pi T$ and not by $m$ when $m / T_{c}<\pi T / T_{c}$.

\subsection{2 $T<T_{c}$}

We also made a series of simulations at fixed $T / T_{c}=0.75$ for $N_{t}=6,8,10$ and 12 in quenched QCD with the Wilson action. We found that the continuum limit of both $\left\langle\mathscr{O}_{2}\right\rangle$ and $\left\langle\mathscr{O}_{11}\right\rangle$ were consistent with zero within reasonably small errors. However, note that at coarser lattices the values were increasing with increasing temperature, but below $T_{c}$ the behaviour is reversed. We then do a dynamical simulation near $T_{c}$ to investigate this further.

\subsection{Dynamical staggered quarks}

We give the results of a series of computations with two flavours of dynamical quarks as the temperature varies between $0.75 T_{c}$ and $2 T_{c}$. The simulations were performed using the R-algorithm with two flavours of quarks with bare mass of $0.1 T_{c}$. Three lattice sizes were used in most of the simulations, namely $4 \times 8^{3}, 4 \times 12^{3}$ and $4 \times 16^{3}$. Lattice computations with dynamical quarks are still too costly for the continuum limit to be taken easily. However, in order to have some idea of the continuum limit we have also performed two further simulations with $6 \times 12^{3}$ and $8 \times 16^{3}$ lattices at $T=0.9 T_{c}$. In addition, we have results at $T_{c}$ on $6 \times 12^{3}$ lattices.

Consistent with our previous observations, $C_{S}^{1}$ was seen to vanish with small errors across the full range of temperatures considered. The diagonal QRC was negative and differed significantly from zero, as shown in Figure 2. Eq. (2.3) then implies the supression of pion fluctuations and increase in pion mass as one approches the critical end point. Note a small shift of the peak from $T_{c}$. This may be an indicator of a crossover transition. For comparison, results from the quenched theory at the same bare quark mass are also shown. The $T$-dependence is seen to be a little different; 


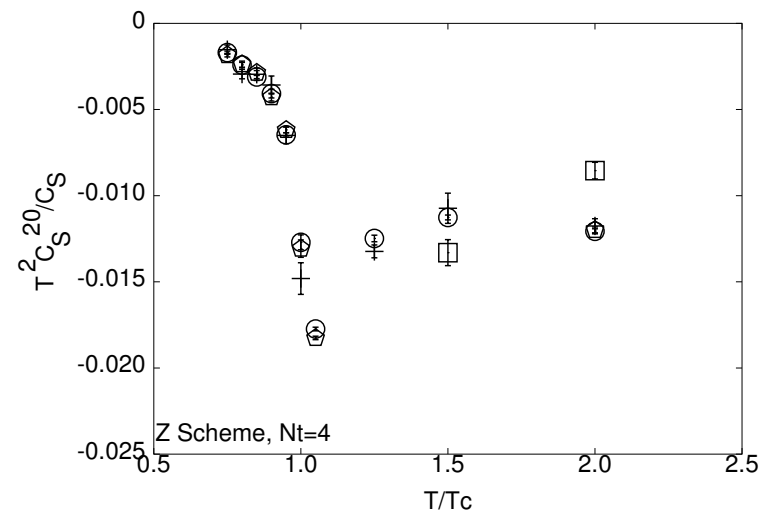

Figure 2: The diagonal QRC as a function of the temperature for $a=1 / 4 T$. Pluses denote results obtained on $4 \times 8^{3}$ lattices, pentagons on $4 \times 12^{3}$ and circles on $4 \times 16^{3}$. The boxes represent the quenched QCD value.

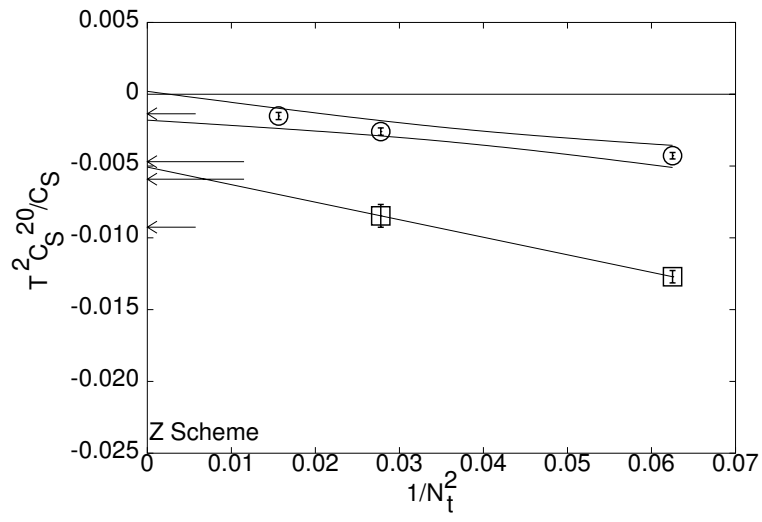

Figure 3: The continuum extrapolation of the diagonal QRC. The circles denote data for $T=0.9 T_{c}$, and the 3$\sigma$ band for the continuum extrapolation is shown. The boxes represent data at $T_{c}$, and the 1- $\sigma$ (nearer arrows) and 3- $\sigma$ (further arrows) limits of the extrapolation are shown.

this could reflect either the difference in the value of $m_{\pi} / m_{\rho}$ or be a quenching artifact. The offdiagonal QRC was consistent with zero within small errors above $T_{c}$. Near and below $T_{c}$ larger fluctuations are seen. Although still consistent with zero at the 3- $\sigma$ level, the averages increase by several orders of magnitude compared to $T>T_{c}$, and seem to be comparable in magnitude to the diagonal QRC. Reduction of the noise in measurement is required to get a clearer signal. Any non-zero value of $C_{S}^{11}$ would imply an assymetry of the phase diagram in the $\mu_{0}$ and $\mu_{3}$ directions (see eq. 2.2)

The results of our preliminary investigation of the continuum limit with dynamical quarks are shown in Figure 3. At $0.9 T_{c}$ the 3- $\sigma$ error band on the extrapolation of the diagonal QRC is consistent with zero. At $T_{c}$ the continuum extrapolation perforce had to be performed with data from only two lattice spacings. With this caveat, our data currently points to a non-zero continuum extrapolation of renormalized $C_{S}^{20}$ at $T_{c}$.

\section{References}

[1] Patel A 1984 Phys. Lett.B 141244

[2] Gupta S 1999 Phys. Rev.D 64094505

[3] Hatta Y and Ikeda T 2003 Phys. Rev.D 67014028

[4] Kogut J B et al.1983 Nucl. Phys.B 22593

[5] Gavai R V and Gupta S 2002 Phys. Rev.D 65094515

[6] Gavai R V and Gupta S 2003 Phys. Rev.D 67034501

[7] Gupta S and Ray R 2004 Phys. Rev.D 70114015 